\title{
Melanom-Prophylaxe: Es bleibt noch viel zu tun
}

\author{
Weltweit nimmt die Inzidenz des malignen Melanoms noch immer zu. In einer \\ dänischen Studie wurde jetzt deutlich, welche Gruppen am stärksten betroffen \\ sind und wo sich Interventionen zur Prävention besonders lohnen könnten.
}

aut Robert Koch-Institut sind seit den _1980er-Jahren die altersstandardisierten Erkrankungsraten für das maligne Melanom in den westlichen Industrieländern stetig angestiegen. Die höchsten Zahlen innerhalb Europas werden aktuell für Dänemark festgestellt, weltweit liegt das Land bei der Melanomhäufigkeit auf Platz 5. Obwohl die allgemeine Krebsrate bei den Dänen stagniert, hat sich die Zahl der Melanompatienten in den vergangenen 25 Jahren mehr als verdoppelt (2012: Männer 29,5 und Frauen 31,7 Erkrankungen pro 100.000 Personenjahre). Damit ist das maligne Melanom in Dänemark die häufigste Krebserkrankung bei jungen Frauen und die zweithäufigste bei Männern zwischen 15 und 39 Jahren. Die 5-Jahres-Überlebensraten zeigen zwar eine positive Tendenz. (2012: Männer 85\% und Frauen $91 \%$ ). Doch während sich die krankheitsspezifische Mortalitätsrate bei den Frauen stabilisiert, steigt sie in der männlichen Bevölkerung weiter. Um solche Entwicklungen besser zu verstehen und ihnen künftig im Vorfeld begegnen zu können, haben Forscher in Dänemark die Daten von Patienten mit invasiven und In-situ-Melanomen aus den Jahren 1985 bis 2012 analysiert.

Insgesamt wurden 3.299 Probanden mit Melanoma in situ und 20.760 Teilnehmer mit malignem Melanom in die Studie aufgenommen. Über die Beobachtungszeit hinweg stieg die Inzidenz des malignen Melanoms bei Männern und Frauen signifikant an. Bei Männern lag die geschätzte prozentuale jährliche Veränderung („estimated annual percentage changes", EAPC) bei $4,5 \%$, bei Frauen erreichte sie $4,3 \%$. Besonders hoch waren die Zunahmen in der Gruppe der über 60-Jährigen (Männer EAPC $5,8 \%$; Frauen $4,8 \%$ ) sowie bei jungen Menschen zwischen 21 und 30 Jahren (EAPC 4,7\% bzw. 6,0\%). Dabei waren Rumpf und Arme am stärksten betroffen und bei den Frauen zudem zunehmend die Schenkel. Hohe EAPCs wur- den auch für Patienten mit einer Tumordicke $<0,75 \mathrm{~mm}(6,6 \%$ bei Männern und $6,1 \%$ bei Frauen) sowie mit superfiziell spreitenden malignen Melanomen (5,2\% bzw. 4,7\%) errechnet. Die relativen Ulzerationsraten dagegen sanken bei beiden Geschlechtern deutlich.

Ein starker Anstieg war auch beim Melanoma in situ erkennbar (EAPC 14,0\% bei Männern und 11,6\% bei Frauen), und zwar in allen Altersgruppen, abgesehen von den 14-20-Jährigen. Besonders deutlich waren auch hier die Zuwächse bei über 60-jährigen Männern sowie bei jungen Frauen zwischen 21 und 30 Jahren (EAPC 14,3\% bzw. 14,2\%).

Fazit: Die Studie bestätigt frühere Befunde über den weltweiten Anstieg der Melanominzidenz. Deutlich erkennbar wurde eine sehr rasche Zunahme in den frühen 2000er-Jahren. Hier kämen möglicherweise bessere diagnostische Maßnahmen sowie die Einführung der Routinedermatoskopie zum Ausdruck, so die Autoren, die sie jedoch nicht für die alleinige Ursache des Anstiegs halten. Die Ergebnisse verdeutlichen ihrer Ansicht nach die Notwenigkeit einer Primärprävention vor allem bei jungen Menschen sowie der gezielten Sekundärprävention bei den Älteren. Denn der relative Anstieg des Melanoma in situ, die häufig geringere Tumordicke, mehr 5-JahresÜberlebende sowie weniger ulzerierende Tumoren sprächen für einen positiven Effekt sekundärpräventiver Bemühungen durch Frühdiagnostik und -therapie. Dass allerdings weitere Anstrengungen in der Primärprävention nötig seien, zeigten unter anderem die noch immer steigenden Mortalitätsraten bei den Männern sowie die weitere Zunahme der Erkrankungen im Bereich sonnenexponierter Körperregionen. Dr. Christine Starostzik

Helvind NM et al. Incidence of In Situ and Invasive Melanoma in Denmark From 1985 Through 2012. A National Database Study of 24059 Melanoma Cases. JAMA Dermatol 2015; 151: 1087-95 